\title{
Determinants of correct res site alignment in site-specific recombination by $\operatorname{Tn} 3$ resolvase
}

\author{
Amy L. Bednarz, Martin R. Boocock, and David J. Sherratt \\ Institute of Genetics, University of Glasgow, Glasgow G11 5JS, Scotland UK
}

In site-specific recombination reactions catalyzed by $\operatorname{Tn} 3$ resolvase, the right and left arms of the res site are always religated to the correct partner. This poses the problem of how resolvase aligns the two sites correctly for the cleavage/religation reaction. We show that the "accessory" binding subsites II and III of res are important for correct alignment of the adjoining crossover subsite (subsite I). Deletion of subsites II and III from one of the two res sites removes a barrier to recombination between incorrectly aligned crossover subsites. Correct alignment does not require any DNA sequence asymmetry in the crossover subsite, DNA supercoiling, or covalent linkage of the two res sites. Our results suggest that correct subsite I alignment is determined by local, resolvase-mediated interactions of subsites II and III of both partners, consistent with a current model of the synapse. Surprisingly, the topological selectivity for intramolecular resolution in a supercoiled substrate does not require subsites II and III in both recombination partners.

[Key Words: Tn3 res/resolvase; site-specific recombination; site alignment; resolution selectivity]

Received June 28, 1990; revised version accepted October 1, 1990.

Site-specific recombination enzymes mediate precise rearrangements of DNA sequences (for review, see Sadowski 1986). They have many different functions, including integration and excision of phage into and out of the bacterial chromosome (Landy 1989), gene switching by inversion of DNA segments (Glasgow et al. 1989), plasmid monomerization and multimerization-processes important for the stable maintenance of plasmids at the correct copy number (Austin et al. 1981; Summers and Sherratt 1984; Futcher 1986) - and resolution of the intermediates of replicative transposition to give the transposition end products (Reed 1981). In vertebrates, programmed DNA rearrangements reminiscent of sitespecific recombination are important in the generation of antibody diversity (e.g., see Lewis and Gellert 1989).

The well-characterized yeast and prokaryote site-specific recombinases fall into two families: the phage $\lambda$ integrase family (Argos et al. 1986) and the resolvase/DNA invertase family (Hatfull and Grindley 1988; Sherratt 1989|. These two families of recombinases are not structurally related, but they share some features that distinguish them from general recombination systems, which act on extended segments of homologous DNA. Site-specific recombinases act at clearly defined DNA recognition sites. Their catalytic mechanism involves synapsis of the two sites, staggered cleavage of the DNA phosphodiester backbone, and religation of the DNA ends to give two recombinant DNA junctions. Resolvases and DNA invertases are thought to cut all four strands of the DNA before any ends are religated, whereas enzymes of the $\lambda$ integrase group initially cut, exchange, and religate one pair of strands to generate a Holliday junction intermediate. During strand exchange the phosphorylated DNA ends are transiently joined to a seryl residue (resolvases/DNA invertases) or tyrosyl residue (integrases) at the catalytic site of the enzyme (for review, see Hatfull and Grindley 1988; Landy 1989 .

Many recombinases catalyze a complete recombination reaction only when the recognition sites are aligned correctly and in a specific topological linkage. Tn 3 resolvase selectively catalyzes an intramolecular resolution reaction (see Fig. 2, below), which is required for the second step of Tn 3 transposition (Reed 1981). The Tn3 res recombination site consists of three discrete binding sites for resolvase; the specialized subsite for strand exchange (subsite I, the crossover subsite) is adjoined by two accessory binding sites (subsites II and III) that are essential for efficient recombination in vivo and in vitro (see Fig. 5, below; Grindley et al. 1982; Kitts et al. 1983; Wells and Grindley 1984). Accessory binding sites for the recombinase or additional protein factors are a characteristic feature of other recombination systems with a stringent topological selectivity (Sadowski 1986). The DNA invertases are an exceptional case; they require two structurally simple crossover sites and a separate cis-acting recombination enhancer element that binds the Escherichia coli FIS protein (Johnson and Simon 1985; Kahmann et al. 1985; Koch and Kahmann 1986). A key objective of current research is to understand how the accessory binding sites and proteins modulate the 
recombination reaction at the crossover sites (Hatfull and Grindley 1988; Klippel et al. 1988; Landy 1989).

In the res/resolvase system and in other microbial recombination systems (but not in the immunoglobulin system|, religation of left ends to right ends is required (Sadowski 1986; Lewis et al. 1988). Distinguishing these ends may not be trivial, as the crossover sites have imperfect dyad symmetry and are believed to bind a symmetrical dimer of recombinase. Thus, one might expect recombinase-mediated synapsis of the crossover sites to be possible in either the "correct" (parallel) or "incorrect" (antiparallel) alignment, potentially giving both correct and incorrect recombinant junctions (see Fig. 1).

How might resolvase ensure correct crossover site alignment during recombination? Recombinases of the integrase family test the alignment of the homologous sites using direct DNA-DNA interactions. To do this, they rely on an asymmetric 6- to 8-bp "overlap" sequence at the center of the crossover site (Hoess et al. 1986). The alignment is not tested during synapsis of the sites but during the strand exchange reaction, in which a Holliday junction migrates through the asymmetric core sequence (de Massy et al. 1989; Nash and Robertson 1989; Nunes-Duby et al. 1989|. The Tn3 res crossover site, however, is cut with only a 2-bp stagger at the center of a perfectly symmetrical hexanucleotide, TTATAA (Reed and Grindley 1981). The right and left arms of the crossover site differ at 6 of 11 positions (see Fig. 5, below). We wished to test whether resolvase recognizes these differences to align the sites correctly. Alternatively, resolvase could recognize the right and left arms of the crossover site indirectly, through the asymmetric location of subsites II and III; specific proteinprotein contacts between different resolvase subunits could be used to align the sites. A third possibility is that the correct alignment is defined indirectly because

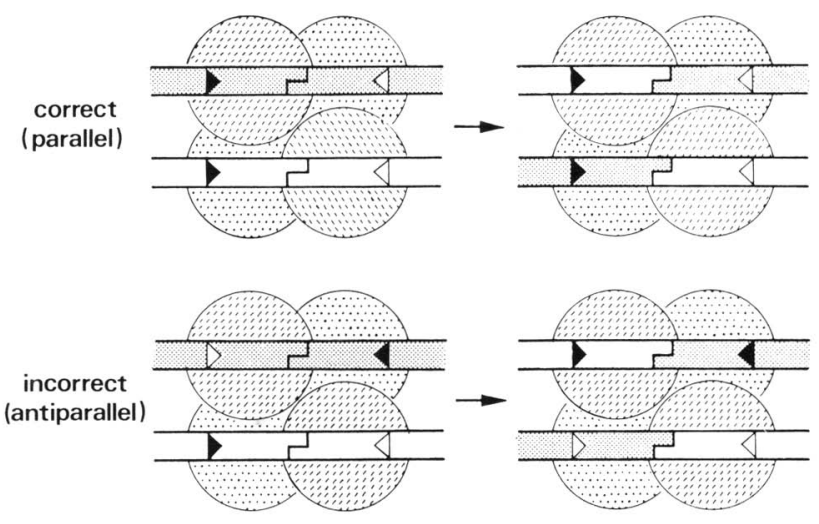

Figure 1. Alignment of crossover sites in site-specific recombination. The cartoons illustrate the synapsis of two crossover sites by protein-protein interactions between the recombinase subunits, with the sequences aligned in a parallel or antiparallel sense, thus giving rise to correct (left-right) or incorrect (leftleft) recombinant junctions. Note that we use correct and incorrect rather than parallel/antiparallel or left-right/left-left; these latter terms are potentially misleading, as they have been used by others to describe different phenomena.

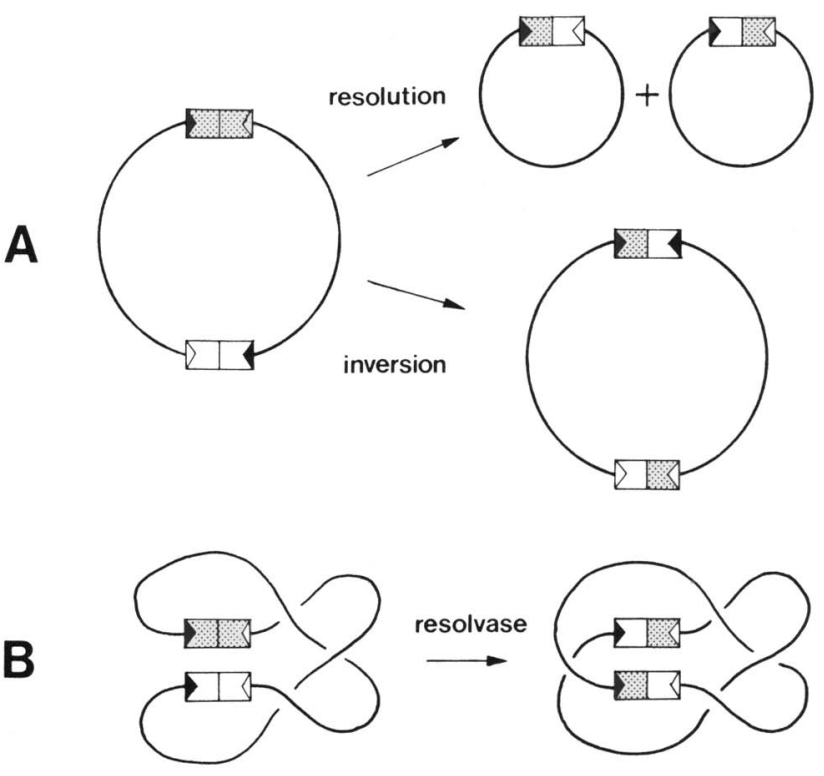

Figure 2. Resolution selectivity in site-specific recombination. $|A|$ Resolution and inversion in a circular substrate containing two recombination sites in direct repeat. If the sites recombine in the parallel alignment, giving correct (left-right) junctions, the substrate is resolved into two circular products. If the sites recombine in the antiparallel alignment, giving incorrect (left-left) junctions, a segment of the substrate is inverted. For a substrate with sites in inverted repeat, these outcomes are reversed. $(B)$ Topology of the resolution reaction between complete res sites in direct repeat /Wasserman and Cozzarelli 1985; Wasserman et al. 1985). Three negative interdomainal supercoils are trapped between the synapsed crossover sites; the recombination product is a simple catenane of two circles with a specific stereochemistry.

the mechanism of synapsis traps exactly three interdomainal supercoils (Fig. 2B).

Resolvase acts efficiently only on directly repeated res sites in cis and not on inverted sites or sites in separate molecules. Recombination between two directly repeated res sites always "resolves" the circular substrate into two circular products (rather than inverting a segmentl, because the crossover subsites are cut and rejoined in the "parallel" alignment (Fig. 2A; Reed 1981). We refer to this behavior as the resolution selectivity of the system. The closely related Hin/Gin/Cin DNA invertases exhibit an analogous inversion selectivity. Contrasting models to account for resolution selectivity emphasize processive "tracking" or "scanning" of resolvase along the DNA (Kitts et al. 1983; Krasnow and Cozzarelli 1983), "slithering" of plectonemically supercoiled DNA (Benjamin and Cozzarelli 1986), and topological restrictions on the formation of an interwrapped synapse (Boocock et al. 1986).

Precisely three interdomainal supercoils are trapped between the crossover sites in the $\operatorname{Tn} 3$ resolution reaction (Fig. 2B; Wasserman and Cozzarelli 1985; Wasserman et al. 1985). A current model suggests that the topological selectivity is associated with a specific plectonemically interwrapped synaptic complex (Boocock et 
al. 1986; Stark et al. 1989). This model predicts an essential role for the accessory subsites II and III of both partners in synapsis, alignment and resolution selectivity (see Fig. 8A, below). To investigate the importance of different regions of res for these processes, we studied the effect of deleting subsites II and III and the effect of replacing the wild-type crossover subsite with a perfectly symmetrical sequence. Our data indicate that subsites II and III of both partners are needed for correct crossover site alignment but are not essential for resolution selectivity.

\section{Results}

Resolvase recognizes the isolated crossover subsite from $\operatorname{Tn} 3$ res

To compare the properties of the complete $\operatorname{Tn} 3$ res site and the isolated res crossover site (subsite I), we deleted subsites II and III using exonuclease III. The sequence of the isolated subsite I and its flanking DNA is shown in Figure 5 (below), aligned with the sequence of the complete res region. Binding of resolvase to the complete res site and to the isolated subsite I was compared using gel retardation assays (Fig. 3; Fried and Crothers 1981). Under our binding conditions, six discrete resolvaseDNA complexes are formed from the complete res site (Fig. 3, lanes 5-8: presumptively two complexes for each subsite; A.L. Bednarz and M.R. Bocock, unpubl.). Two complexes are obtained from the isolated subsite I (Fig. 3, lanes 1-4). Full occupation of the isolated subsite I occurs at resolvase concentrations comparable to those required for full occupation of the complete res site (Fig.

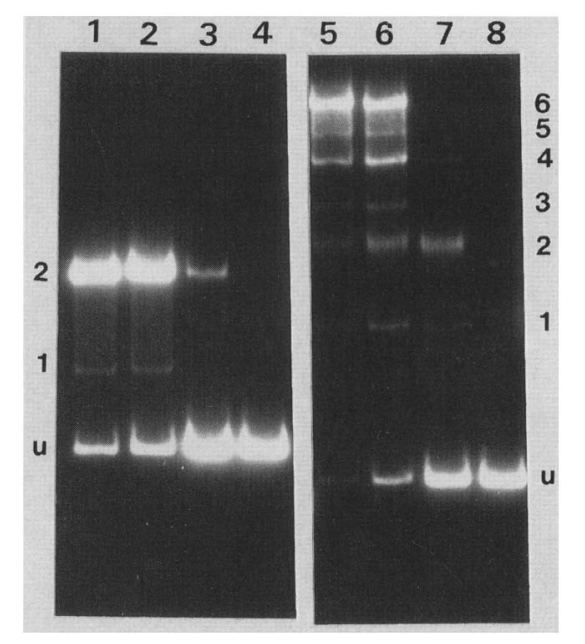

Figure 3. Binding of resolvase to subsite I and to the complete res site. Gel retardation assays used a 203-bp EcoRI fragment carrying subsite I of res (lanes 1-4) and a 168-bp BamHI-EcoRI fragment carrying the complete res site (lanes 5-8). Fragments labeled with ${ }^{32} \mathrm{P}$ were incubated with resolvase as described in Materials and methods; the protein-DNA complexes were separated on a $6 \%$ nondenaturing polyacrylamide gel and are designated 1-6 (vertically). (u) The unbound DNA fragment. (Lanes 1-5) $112 \mathrm{nM}$ resolvase; (lanes 2-6) $56 \mathrm{nM}$ resolvase; (lanes 3 and 7) $28 \mathrm{nM}$ resolvase; (lanes 4 and 8) no resolvase.

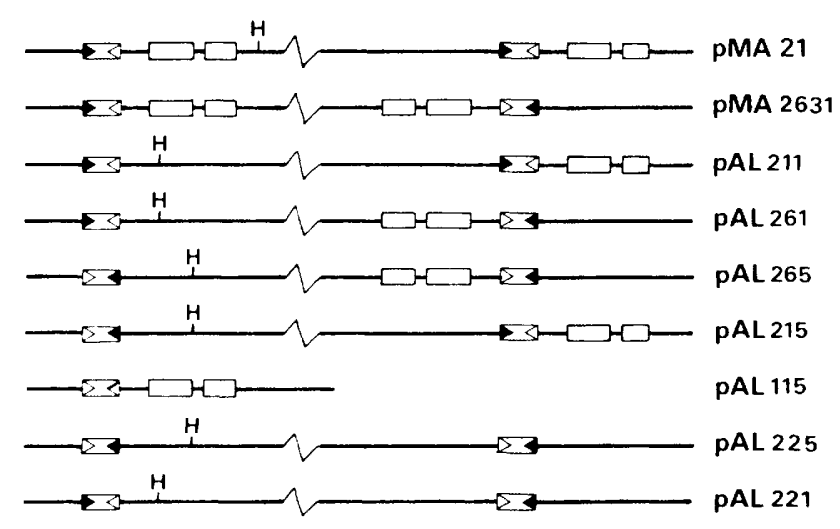

Figure 4. Arrangement of res sites in the substrates used for intramolecular recombination. Subsites I, II, and III of res are represented as rectangular boxes (for DNA sequences, see Fig. 5). The open and solid arrowheads represent the right and left arms of subsite I, respectively. The pBR322 vector sequences are not shown to scale. $(\mathrm{H})$ HindIII site. The constructions are detailed in Materials and methods.

3). Subsites II and III are therefore not necessary for avid binding of resolvase at subsite I.

\section{Recombination between subsite I and the complete res site}

To test the recombination properties of an isolated subsite I, we constructed a family of pBR322-derived substrates containing the complete res site and the isolated subsite I in different combinations and configurations (Fig. 4). Recombination was assayed in vitro using negatively supercoiled plasmid substrates, purified Tn3 resolvase, and reaction conditions that favor the recombination of poor substrates (buffer A or B; Materials and methods). A substrate containing two intact res sites in direct repeat, pMA21, resolved to completion within 1 hr. Under the same conditions, we observed slow recombination between the isolated subsite I and a complete res site in direct repeat, using the substrate pAL211 $(<20 \%$ product in $19 \mathrm{hr}$; Fig. $6 \mathrm{~A}$, below). We estimate that the rate of recombination is $<1 \%$ of that for the reaction between two complete res sites. Recombination between two copies of the isolated subsite I, either in direct repeat (pAL225) or in inverted repeat (pAL221), was not detectable in supercoiled substrates under any reaction conditions tested. Thus, subsites II and III greatly stimulate in vitro recombination between the crossover sites but are not essential in both recombination partners.

We also attempted to recombine a substrate, pAL215, that contains an isolated subsite I and a complete res site in inverted repeat (Fig. 4). pAL215 recombined in vitro with a similar efficiency to pAL211 (subsite I and complete res in direct repeat). Restriction analysis after these reactions revealed only resolution products (Fig. 6B, below) and no detectable level of inversion products $k 1 \%$ of total product). Thus, the selectivity for resolution over inversion is maintained in recombination reac- 
tions between subsite I and the complete res, regardless of the relative orientation of the two sites in the substrate.

In the resolution reaction of pAL215, the right arm of the isolated subsite I was religated to the right arm of the complete res site. The circular plasmid containing this recombinant junction was isolated from the reaction products. DNA sequencing of the junction revealed a novel recombinant res site with a perfectly symmetrical subsite I, as expected if the two crossover sites are cleaved at the usual positions and the two right arms are religated to each other. This site was called sym-res (Fig. 5). (The junction between the two left arms was not sequenced because it could not be maintained stably in vivo; the predicted junction sequence is a 300 -bp palindrome, longer than can be stably maintained in a rec $A$ strain; see Boissy and Astell 1985). The structure of the resolution products of pAL215 is thus indicative of incorrect alignment of the crossover sites during strand exchange (Fig. 1). Note that our assay for site alignment requires the formation and analysis of recombinant products; incorrectly aligned sites that fail to recombine will not be detected.

To check that the vector DNA sequences that replace subsites II and III do not affect the alignment or reactivity of the isolated subsite I, we compared four substrates with the recombination site cassettes inserted in different configurations: pAL211, pAL261, pAL265, and pAL215 (Fig. 4). In these substrates the left arm of subsite I adjoins the natural upstream sequence from $\operatorname{Tn} 3$, while the right arm adjoins one of two different vector sequences (Fig. 5). The four substrates all recombined with a similar low efficiency, and all four gave resolution products and no detectable inversion products. Correct junctions were formed when the two recombination sites were in direct repeat (pAL211 and pAL265); incorrect junctions were formed when the sites were in inverted repeat (pAL261 and pAL215). We conclude that the alignment of the crossover sites in the cleavage/religation reaction is determined solely by the relative orientation of the subsite I and the complete res within the substrate. The differences between the left and right arms of subsite I and their different flanking sequences did not determine which arm of subsite I was religated to the left arm of the complete res. Three different subsite I flanking sequences substituted equally inefficiently for the deleted subsites II and III in the resolution reactions of pAL211, pAL265, and pAL261 (Fig. 5). Because these sequences do not resemble each other, or the deleted res sequences, we conclude that the sequence of this flanking DNA is not important for the resolution reaction. Using a 203-bp DNA fragment containing the isolated subsite I cassette in the gel-binding assay, we were unable to detect any additional complexes attributable to specific binding of resolvase to the flanking sequences (Fig. 3).

To test whether truncation of the accessory subsites is needed to allow recombination with the incorrect alignment of the crossover subsites, we compared the recombination properties of pMA2631 (two complete res sites in inverted repeat) and pAL261 (one site truncated). Using supercoiled pMA2631, we failed to detect any products of a resolution reaction between the res sites under conditions that gave up to $20 \%$ resolution of pAL261 (Fig. 6A). Thus, the second set of accessory sites in pMA2631 appears to block the resolution reaction between the crossover subsites. This contrasts with the result obtained for res sites in direct repeat; the second set of accessory sites greatly stimulated the resolution reaction (e.g., in pMA21, compared with pAL211). Inversion reactions of the supercoiled pMA2631 substrate were also undetectable, consistent with previous observations that inverted sites recombine only in nonsupercoiled substrates (Boocock et al. 1987; J.L. Brown et al., unpubl.) or in knotted supercoiled substrates (Droge and Cozzarelli 1989).

Our results for in vitro recombination of supercoiled substrates are summarized in Table 1. Recombination between two isolated crossover sites was not detectable. When one of the crossover sites was adjoined by subsites II and III, resolution proceeded slowly, and inversion was not detectable, regardless of the relative orientation of

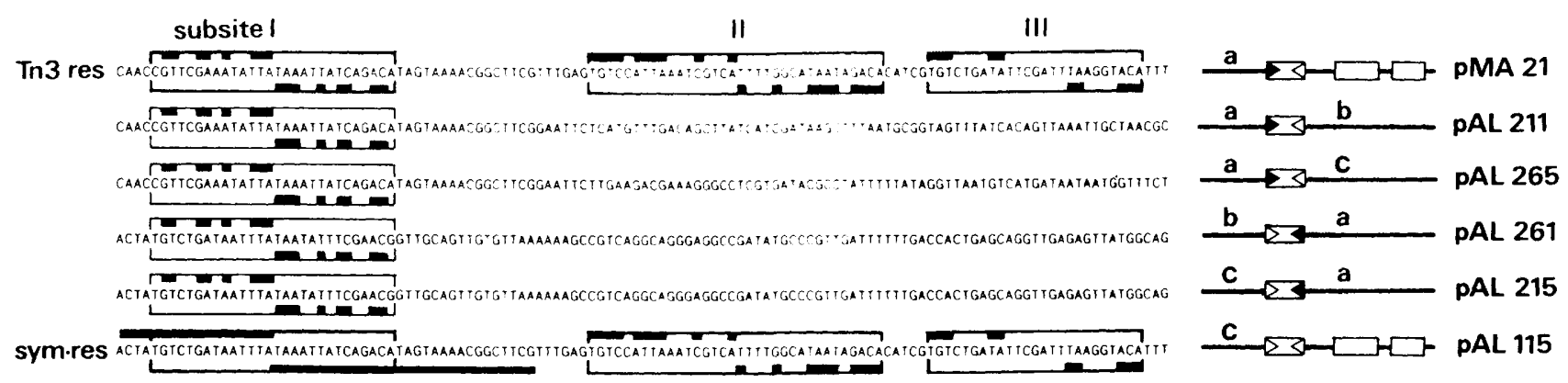

Figure 5. Sequence of the complete Tn 3 res site, the isolated subsite I and flanking sequences, and the sym-res site. Bold lines indicate the partial dyad symmetry of each subsite. The flanking sequences shown to the right of subsite I are the sequences that would need to substitute for subsites II and III in a resolution reaction with the complete res site, in the substrate indicated. The sequences are presented in the same orientation as the complete res site in that substrate; the subsite I sequence therefore appears in the reverse orientation for pAL261 and pAL215. The cartons at right clarify the relationships between the flanking sequences, designated $a, b$, and $c$, on both sides of subsite I. Subsites I, II, and III of res are shown as in Fig. 4 . The wild-type Tn3 res and pBR322 sequences are taken from Heffron and McCarthy (1979) and Sutcliffe (1979). 
Figure 6. In vitro recombination between subsite I and a complete res in supercoiled substrates. Substrates were incubated with resolvase in buffer A (gel $A$ ) or buffer $\mathrm{B}$ (gels $B, C$, and $D$ ) containing 25 $\mathrm{mm} \mathrm{NaCl}$, at $37^{\circ} \mathrm{C}$ for $19 \mathrm{hr}$ (except gel $C$; $16 \mathrm{hr}$. Reaction products were analyzed on $1.2 \%(A-C)$ or $0.7 \%(D)$ agarose gels. (A) Restriction analysis of reaction products from pMA21, pMA2631, pAL211, and pAL261 (see Fig. 4). Reactions contained $0,200,400$, and $800 \mathrm{~nm}$ resolvase (lanes $a-d$ ). All samples were cut with PstI and HindIII. (S) Restriction fragments corresponding to unrecombined substrate; $(\mathrm{P})$ restriction fragments with sizes corresponding to resolution products; (m) size markers 3234, 2817, 2466 , and $1129 \mathrm{bp}$. The small amounts of inversion product obtained with pMA2631 (lanes $b-d$ with pMA2631; restriction fragments of a similar size would be diagnostic for inversion of pMA2l, pAL211, pAL261, pAL215, and pAL265) are due to traces of nicked substrate in the reaction. $(B)$ Characterization of resolution products from pAL215 and pMA21. Reactions contained $0,50,100$, and 200 nM resolvase $(0,+,++$, and +++1 . Products were cut with either Pst $(\mathrm{P})$ and/
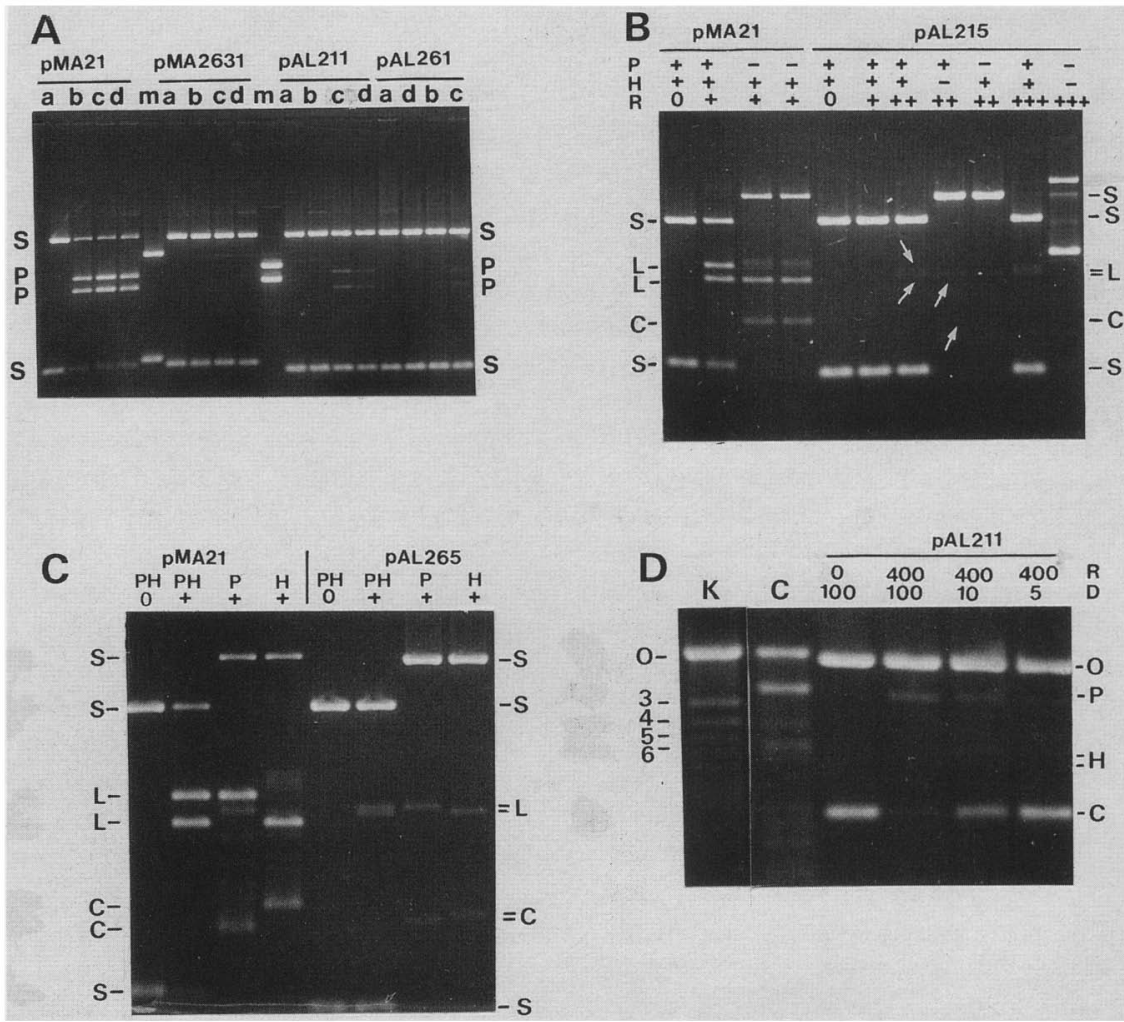
or HindIII $(\mathrm{H})$ to reveal supercoiled cir-

cular resolution products $(\mathrm{C})$ and the corresponding linear DNA fragments $(\mathrm{L})$. The free supercoiled circles were not detectable in the uncut products of pAL215 recombination; this was confirmed by Southern hybridization of similar gels. $(C)$ Characterization of resolution products from pAL265 and pMA21. Reactions contained $400 \mathrm{nM}$ (pAL265) or $200 \mathrm{nM}$ (pMA21) resolvase (+1, or no resolvase (0). Products were cut with PstI (P) and/or HindIII (H), as indicated. (S) Restriction fragments corresponding to unrecombined substrate; $(C)$ supercoiled circular resolution products; $(\mathrm{L})$ linear restriction fragments corresponding to circular resolution products. $|D|$ Topological analysis of resolution products of pAL211. Reactions contained 0 or $400 \mathrm{nM}$ resolvase, as shown; the products were nicked with 5,10 , or $100 \mathrm{ng} / \mathrm{ml}$ DNase I. Marker tracks contain nicked knots $(\mathrm{K})$ and simple catenanes $(\mathrm{C})$ made from pMA21 using T4 topoisomerase and resolvase, respectively. $(\mathrm{C})$ Supercoiled pAL211; $(\mathrm{O})$ unknotted nicked circles; $(\mathrm{P})$ nicked resolution product; $(\mathrm{H})$ half-nicked resolution products.

the two sites. In substrates containing two complete res sites in direct repeat, resolution proceeded efficiently; when the sites were in inverted repeat, neither resolution nor inversion was detectable.

The isolated $\operatorname{Tn} 3$ res crossover site did not recombine with a complete res site at a significant rate when complemented in vivo with a resolvase-overproducing plasmid (A.L. Bednarz and D.G. Blake, unpubl.) in agreement with previous results for $\gamma \delta$ res (Wells and Grindley 1984).

\section{Topology of recombination between subsite I and complete res site}

To investigate the effect of deleting subsites II and III from one partner on the topology of recombination, we analyzed the topology of the resolution products. Restriction with the appropriate enzymes revealed that both circular products are highly supercoiled (Fig. 6C, lanes 7 and 8 ). The circles were also exclusively catenated, as they were only released after restriction

Table 1. Summary of intramolecular recombination reactions

\begin{tabular}{lccccc}
\hline Substrate & Sites & Orientation & $\begin{array}{l}\text { Resolution } \\
\text { efficiency }\end{array}$ & $\begin{array}{l}\text { Inversion } \\
\text { efficiency }\end{array}$ & $\begin{array}{c}\text { Alignment of } \\
\text { sites (resolution) }\end{array}$ \\
\hline pMA21 & res + res & direct & 100 & $<0.1$ & correct \\
MA2631 & res + res & inverted & $<0.1$ & $<0.1$ & - \\
pAL211, pAL265 & res + subsite I & direct & 1 & $<0.1$ & correct \\
pAL215, pAL261 & res + subsite I & inverted & 1 & $<0.1$ & incorrect \\
pAL225 & subsite I + subsite I & direct & $<0.1$ & $<0.1$ & - \\
pAL221 & subsite I + subsite I & inverted & $<0.1$ & $<0.1$ & - \\
\hline
\end{tabular}


cleavage (Fig. 6B, lanes 8, 9, and 11); Electrophoresis of uncut reaction products revealed a species with a mobility slightly greater than the supercoiled substrate, as expected for a supercoiled catenane (not shown). To analyze the complexity of the catenanes, we nicked the reaction products with DNase I and compared the mobilities of the nicked species with a reference ladder of knots made with T4 topoisomerase II (Spengler et al. 1985). The major nicked product had the mobility of a simple two-noded catenane; significant amounts of unlinked circles or complex catenanes were not detectable (Fig. 6D). Thus, the resolution products were simple catenanes of supercoiled circles, as in the reaction between two complete res sites. This indicates that recombination between subsite I and a complete res is a concerted reaction in which all four DNA strands are cut and religated without releasing most of the supercoiling. Also, the topology of productive synapsis and strand exchange is strictly controlled, as it is in the standard reaction (Wasserman and Cozzarelli 1985).

\section{Recombination properties of the sym-res site}

The results described above indicate that resolvase fails to distinguish the left and right arms of subsite I when subsites II and III have been deleted and therefore fails to align the crossover sites correctly. To test whether the accessory subsites II and III are sufficient to direct correct alignment of the crossover sites, we investigated the recombination reactions of sym-res, a res site with a perfectly symmetrical crossover subsite. Plasmid pAL115 was obtained by in vitro resolution of pAL215; it carries the sym-res site, in which the wild-type subsite $I$ is replaced by a perfectly symmetrical 60-bp sequence consisting of two right arms of subsite I and adjacent res sequences (Fig. 5). A recombination substrate with two sym-res sites in direct repeat was made by isolating a dimer of pAL115. Supercoiled pAL115 dimer recombined in vitro with a similar efficiency to pMA21 (two wild-type res sites). The recombination products from the pAL115 dimer were catenated circles, and restriction analysis revealed no detectable amount of any inversion products indicative of recombination between incorrectly aligned crossover sites (not shown). The dimer plasmid was also resolved efficiently in vivo, when complemented with a $\operatorname{tn} p R^{+}$plasmid in a recA background. These results clearly indicate that the asymmetry of the wild-type subsite I sequence is not necessary for correct alignment and resolution selectivity or for efficient recombination.

We were concerned that the 60 -bp palindrome in sym-res might extrude into a cruciform at in vitro levels of superhelicity and inhibit recombination, as has been reported for symmetrized recombination sites in the lox/Cre system (Hoess et al. 1986). We obtained no evidence of such inhibition with the pAL115 dimer.

To test whether subsites II and III are able to direct a particular alignment of two sym-res sites that are topologically unlinked, we investigated the intermolecular recombination reaction between two linear DNA sub- strates carrying a single copy of sym-res. The substrates used were PstI-linearized pAL145 and PvuII-linearized pAL115; two control substrates carrying a single wildtype res site were made by linearizing pMA2856 with HindIII and SstI. For both pairs of substrates, the major recombination products were linear molecules of the sizes predicted for correct alignment of the crossover sites during strand exchange (Fig. 7). For both pairs of substrates very small amounts of products $<10 \%$ of total recombinant) were detected with the sizes predicted for incorrect alignment of the crossover sites. Thus, the correct alignment of the crossover sites is strongly favored in intermolecular recombination reactions of sym-res, as was observed previously with the wild-type res site (J.L. Brown et al., unpubl.). This result demonstrates conclusively that subsites II and III can define the functional left and right arms of an adjoining crossover site that lacks any internal asymmetry. The very minor products attributable to incorrect crossover site alignment have not been observed previously in reactions with complete res sites and have not been characterized further.

\section{Discussion \\ Alignment of the crossover subsites}

Our results implicate subsites II and III of res in the alignment of the crossover subsites. The experiments on intramolecular recombination in supercoiled substrates showed that an isolated subsite I can recombine, at a reduced rate, with a complete res site (subsites I, II, and III). The remaining subsites II and III were essential for detectable recombination but did not ensure correct alignment of the subsites I. In the reaction between a complete res site and a subsite I in inverted repeat, the incorrect alignment of crossover subsites was used exclusively. The deletion of subsites II and III from one partner thus reduces the overall efficiency of recombination and permits incorrect as well as correct alignment of the subsite I sequences in the cleavage/religation reaction.

The intermolecular recombination reactions between sym-res sites on linear substrates showed that two res sites can be aligned correctly when a region of $60 \mathrm{bp}$ around subsite I has a perfectly symmetrical sequence. This result implies that the left and right arms of subsite I are recognized indirectly and identifies subsites II and III of both partners as the sequences required for this indirect recognition. Our data are consistent with the idea that the crossover site alignment is defined by resolvasemediated interwrapping of subsites II and III of both partners during synapsis (Fig. 8A). (We cannot formally exclude the possibility that the alignment is sensed during the cleavage/religation reaction, but we cannot imagine how this would operate with symmetric crossover subsites.) Further work is needed to determine how the accessory subsites interact in the synapse and to elucidate the role of the individual subsites.

Substrate supercoiling and topological linkage were not required for correct alignment of two complete res 


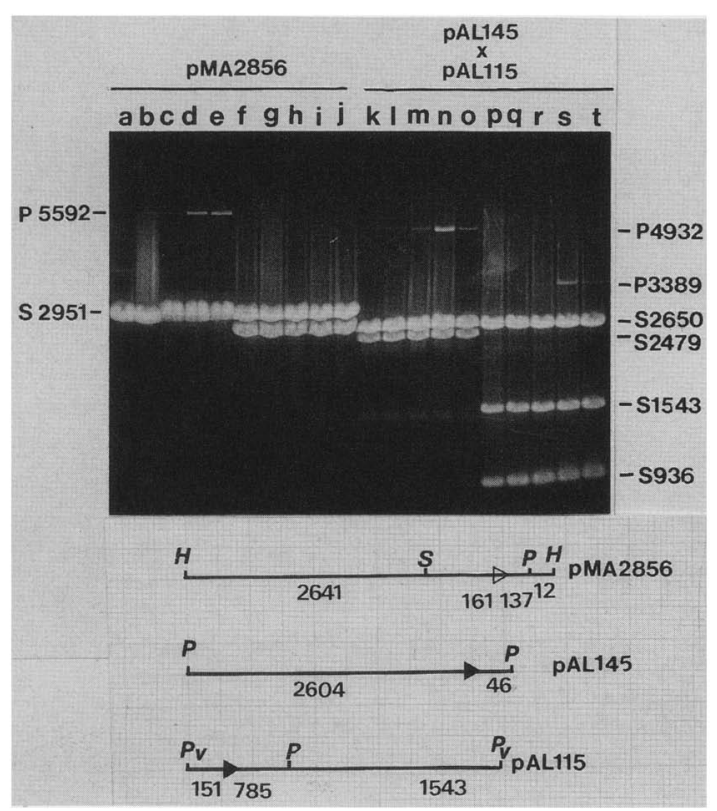

Figure 7. Intermolecular recombination between two linear substrates carrying sym-res. PstI-linearized pAL145 (2650 bp) and PvuI-linearized pALl15 (2479 bp) were incubated with resolvase in buffer $\mathrm{C}+50 \mathrm{mM} \mathrm{NaCl}$ for $22 \mathrm{hr}$ at $37^{\circ} \mathrm{C}$. As a control, two linear substrates carrying the wild-type res site (pMA2856 linearized with either HindIII or SstI, 2951 bp) were treated similarly with resolvase. Recombination products were separated on a $1.2 \%$ agarose gel uncut (lanes $a-e$ and $k-o$ ) or after cleavage with PStI (lanes $f-j$ and $p-t$ ). Resolvase concentrations used were $0,139,278,556$, and $1112 \mathrm{~nm}$ in lanes $a-e$, $f-j, k-o$, and $p-t$. In each of these pairs of reactions, the plasmid substrate could recombine either with its partner or itself, in each of two alignments. There are therefore six possible products from each reaction (three for correct and three for incorrect alignment). Calculations of the expected sizes of substrate, as well as recombinant product, showed that DNA fragments of 5604 bp (pMA2856) and 5208 bp (pAL145 × pAL115), resistant to cleavage by PstI, would be unambiguously diagnostic of incorrect alignment. DNA fragments corresponding to unrecombined substrate are marked S. Reaction products with the sizes predicted for correct alignment of the res sites are marked P; the 4932-bp product of the sym-res reaction yielded a fragment of $3389 \mathrm{bp}$, consistent with the expected structure. Similarly, the 5592-bp fragment resulting from recombination between wild-type res sites was cleaved by $P$ stI to give products of 2939 and 2653 bp. (Below) Outline plasmid maps: (P) Psti; (Pv) PvuII; (H) HindIII. Open arrowhead indicates wild-type res; solid arrowhead indicates sym-res. The bases of the arrowheads represent parts of res containing subsites II and III. The numbers indicate base pairs between restriction sites or restriction sites and the crossover site.

sites (sym-res or wild-type res). This indicates that assembly of the synapse and alignment of the sites does not need to be directed by the supercoil topology of the substrate. Supercoiling evidently stabilizes the synaptic structures (Benjamin and Cozzarelli 1988).

In its reactions with a complete res site, subsite I functioned as if it were perfectly symmetrical; resolvase disregarded the six sequence differences between the left and right arms of subsite I. This is consistent with the idea that subsite I binds a symmetrical dimer of resolvase and synapses and recombines with a second subsite I using D2-symmetric protein-protein contacts (Stark et al. 1989).

Although subsite I is functionally symmetric, it did not recombine with a complete res site with a random alignment of the crossover sites. Only resolution products were obtained; inversion products, diagnostic for the opposite alignment of the crossover sites, were not detected-even when the sites were in inverted repeat. In these reactions, the topological alignment of the crossover sites in the synapse therefore takes precedence over the parallel or antiparallel alignment of their sequences. There is evidence that an analogous mechanism operates in the alignment of crossover sites by the DNA invertases (Kanaar et al. 1990). In the DNA invertase systems, however, the incorrect alignment of crossover site sequences does not lead to recombinant cleavage/religation products, because the sites have an asymmetric core.

In phage $\lambda$ integrative recombination, accessory binding sites flanking the asymmetric att $P$ crossover site determine which arm of this site initiates the strand exchange reaction with attB (Kitts and Nash 1988). In contrast, we have shown that the crossover subsite of Tn 3 res is functionally symmetric and that the adjoining accessory subsites control the alignment of the crossover subsites in the cleavage/religation reaction. Recombination of incorrectly aligned res sites in vivo would generate a lethal palindrome and destroy the transposon. Our data imply that the correct alignment can be selected accurately during synapsis of the crossover sites. Thus, a mechanism for sensing the alignment during strand exchange is probably not needed, as it is in the integrase and DNA invertase systems.

\section{Resolution selectivity}

Surprisingly, resolution selectivity was maintained in intramolecular recombination reactions between an isolated subsite I and a complete res site in either direct or inverted repeat. Resolution selectivity therefore does

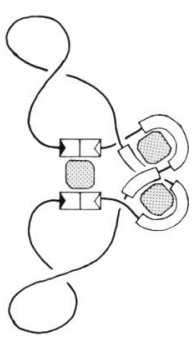

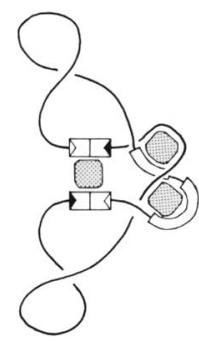

B

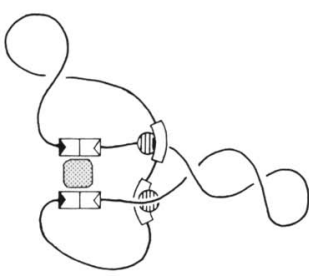

Figure 8. Models of the synaptic structures formed in the following reactions: $(A \mid$ Resolution between two complete res sites in direct repeat; $|B|$ resolution between subsite I and a complete res site in inverted repeat; $(C)$ inversion mediated by DNA invertases. The crossover sites are represented as boxes with arrowheads, the accessory sites as open boxes, resolvase and invertase as shaded squares, and FIS protein as striped circles. 
not require accessory binding sites in both recombination partners or correct alignment of the crossover sites. Deletion of subsites II and III from one partner did not alter the topology of the recombination products; they were supercoiled simple catenanes. Our data strongly suggest that three interdomainal supercoils are trapped, as in the reaction between two complete res sites. This appears to contradict the idea that the three interdomainal supercoils are trapped by interwrapping of subsites II and III of both partners (Fig. 8A). Nevertheless, the formation of an interwrapped structure between two complete res sites is supported by a number of independent experiments (Boocock et al. 1987; Stark et al. 1989; Benjamin and Cozzarelli 1990). We therefore propose that in the reaction between subsite I and a complete res, nonspecific DNA sequences from the supercoiled substrate can substitute inefficiently for subsites II and III of one res site. Critically, this model accounts for the selectively incorrect alignment of the crossover subsites in the reaction between subsite I and a complete res in inverted repeat (Fig. 8B). Direct characterization of the sequences within the synapse will be needed to test this explanation.

We are intrigued by a possible parallel between resolution selectivity in the reaction between subsite I and complete res, and inversion selectivity in the DNA invertase systems. Both systems control the number of interdomainal supercoils, -2 in the inversion reaction (Kanaar et al. 1989) and (by inference) -3 in the resolution reaction, but do not control the alignment of the crossover sites during synapsis. (Kanaar et al. 1990). Both reactions involve two crossover sites and two accessory sites: subsites II and III of res, or the two FIS-binding sites of the recombination enhancer sis. We speculate that in both bases, the synapse contains protein bridges between accessory binding sites and nonspecific DNA sequences (Fig. 8B,C). We suggest that these proteinDNA interactions at the accessory sites organize a local condensed structure that can stimulate recombination selectively between crossover sites in a specific topological alignment. It is possible that the proteins bound at the accessory sites do not need to make direct or specific contact with the recombinase at the crossover sites to stimulate the cleavage/religation reaction. In support of this idea, we note that the spacer between subsites I and II of res can be lengthened by at least two complete helical turns of DNA (Salvo and Grindley 1988). It will therefore be interesting to see whether the accessory sites and proteins can stimulate recombination in the heterologous system.

\section{Materials and methods}

Bacterial strains and plasmids

In vivo recombination was assayed in an Escherichia coli recA strain DS902 (Stark et al. 1989). Dimers of plasmids were made using the $r e c B C s b c A$ strain JC8679 |Summers and Sherratt 1984) to multimerize the plasmid, followed by transformation of DS902 with the reisolated plasmid DNA. res ${ }^{+}$substrate plasmids were generally derived from pBR322 (Sutcliffe et al.
1979|; resolvase was supplied by a compatible pACYC184 derivative, pPAK316.

Subsite I of res was obtained from the complete res site by unidirectional exonuclease III digestion of a res ${ }^{+}$pUC1 8 derivative (Henikoff 1984; Yanisch-Perron et al. 1985). A 203-bp EcoRI fragment from the resulting deletant, pAL3054, was inserted into the EcoRI site of pUC18 in tandem repeat, from whence the deletion endpoint was determined by DNA sequencing. Plasmid pMA21 (4927 bp) was obtained from pBR322 by insertion of 282-bp EcoRI and PvuIl fragments carrying Tn 3 res into the EcoRI and PvuII sites, respectively, with the res sites in direct repeat. pMA2631 is identical to pMA21 except that the res site at the PvuII site is in the reverse orientation. Plasmids pAL211, pAL215, pAL261, and pAL265 (4848 bp) were constructed by inserting the 203-bp EcoRI subsite I fragment from pAL3054 into the EcoRI site of pBR322, in the required orientation, followed by a PstI-AvaI fragment exchange with pBR322 derivatives carrying the complete res as a 282-bp $P_{v u I I}$ insert at the PvuII site. Resolution of pAL215 gave a circular product, pAL115 $(2479 \mathrm{bp} /$, containing the sym-res site. pALl45 (2650 bp) was made by inserting the 145-bp EcoRI sym-res fragment from pAL115 into the EcoRI site of pMTL23, a pBR322-derived polylinker vector obtained from the CAMR laboratory (Porton Down, UK). Plasmid pMA2856 (2951 bp) is the resolution product of pMA2356 (Stark et al. 1989) and carries a 282-bp res insert between the SmaI and HincII sites of pUC18.

\section{End labeling of DNA fragments}

DNA restriction fragments were isolated by elution from polyacrylamide gels and end-labeled using Klenow fragment or T4 polynucleotide kinase. Labeled fragments were purified by phenol and chloroform extractions and precipitated with ethanol.

\section{Gel-binding assays}

A 168-bp BamHI-EcoRI fragment from pMA2350 (complete res) and a 203-bp EcoRI fragment from pAL3054 (subsite I) were labeled for the binding assays. Resolvase was purified as in Stark et al. (1989), diluted in a buffer containing $1 \mathrm{M} \mathrm{NaCl}, 50$ $\mathrm{mM}$ Tris- $\mathrm{HCl}(\mathrm{pH} 8.2)$, and $1 \mathrm{mM}$ EDTA. Binding reactions contained $10 \mathrm{~mm}$ Tris-glycine ( $\mathrm{pH} 9.4) ; 0.1 \mathrm{~mm}$ EDTA, $10 \%$ glycerol ( $\mathrm{vol} / \mathrm{vol}), \sim 1 \mathrm{nM}$ end-labeled DNA fragment, and $20 \mu \mathrm{g} / \mathrm{ml}$ supercoiled pUC18 carrier DNA, in a total volume of $10 \mu \mathrm{l}$, and were initiated by adding $0.4 \mu \mathrm{l}$ of diluted resolvase. The same volume of resolvase dilution buffer was added to reaction blanks. After mixing thoroughly, the reactions were incubated at $37^{\circ} \mathrm{C}$ for $10 \mathrm{~min}$, transferred to ice, and loaded onto the gel almost immediately. Protein-DNA complexes were separated on $6 \%$ polyacrylamide gels containing $50 \mathrm{~mm}$ Tris-glycine $1 \mathrm{pH}$ $9.4), 0.1 \mathrm{mM}$ EDTA. Gels were prerun in the same buffer at 15 $\mathrm{V} / \mathrm{cm}$ for $30-90 \mathrm{~min}$ at $4^{\circ} \mathrm{C}$ and run for $2-3 \mathrm{hr}$ at $15 \mathrm{~V} / \mathrm{cm}$ at $4^{\circ} \mathrm{C}$. Gels were dried onto filter paper and autoradiographed against Kodak X-Omat $\mathrm{S}$ film for $1-3$ days. The resolvase concentration required for a particular extent of binding to the complete res or to subsite I was essentially independent of the concentration of the labeled DNA fragment under these conditions.

\section{In vitro recombination assays}

Recombination reactions contained $\sim 20 \mu \mathrm{g} / \mathrm{ml}$ DNA substrate in one of the following recombination buffers: (A) $50 \mathrm{~mm}$ Tris$\mathrm{HCl}(\mathrm{pH} 8.2), 10 \mathrm{~mm} \mathrm{MgCl}, 0.1 \mathrm{mM}$ EDTA, $5 \mathrm{~mm}$ spermidine- 
$3 \mathrm{HCl}, 20 \%$ glycerol (vol/vol); (B) $50 \mathrm{~mm}$ Tris- $\mathrm{HCl}(\mathrm{pH} 9.4), 10$ $\mathrm{mM} \mathrm{MgCl}_{2}, 0.1 \mathrm{mM}$ EDTA, 20\% glycerol (vol/vol); $(\mathrm{C}) 50 \mathrm{~mm}$ Tris-glycine (pH 9.4), $5 \mathrm{~mm} \mathrm{MgCl}_{2}, 0.1 \mathrm{~mm}$ EDTA, $5 \mathrm{~mm}$ spermidine-trihydrochloride, $20 \%$ glycerol ( $\mathrm{vol} / \mathrm{vol})$. Buffers A and $C$ have been shown to support recombination of nonsupercoiled substrates (Boocock et al. 1987). Reactions were initiated by adding one-twentieth or one-fortieth of the volume of resolvase (prediluted in the same buffer as for binding reactions) to give a final $\mathrm{NaCl}$ concentration of 50 or $25 \mathrm{~mm}$. Resolvase was purified as in Stark et al. (1989). Reactions were incubated at $37^{\circ} \mathrm{C}$ for up to $22 \mathrm{hr}$ and stopped by heating to $70^{\circ} \mathrm{C}$ for $5 \mathrm{~min}$. Samples were then treated with DNase I or restriction enzymes, as required. Sucrose loading buffer containing protease $\mathrm{K}$ was added prior to electrophoresis of samples on $1.2 \%$ and $0.7 \%$ agarose gels made up in $20 \mathrm{~mm} \mathrm{NaOAc}, 1 \mathrm{~mm}$ EDTA, 40 mM Tris-AcOH (pH 8.2). Gels were run horizontally at $\sim 3$ $\mathrm{V} / \mathrm{cm}$, stained with ethidium bromide, and photographed onto Ilford HP5 film using short-wave UV illumination and a red filter.

\section{DNase I nicking of DNA}

DNase I was diluted in $10 \mathrm{mM}$ Tris- $\mathrm{HCl}(\mathrm{pH} 8.0 \mid, 10 \mathrm{~mm} \mathrm{MgCl}$, and $50 \%$ glycerol $(\mathrm{vol} / \mathrm{vol})$ and stored at $-20^{\circ} \mathrm{C}$. Recombination reaction samples in recombination buffer $\mathrm{A}$ or $\mathrm{C}$ were treated with $5-100 \mathrm{ng} / \mathrm{ml}$ DNase I for $10 \mathrm{~min}$ at $0^{\circ} \mathrm{C}$. Samples were then mixed with loading buffer containing $0.2 \%$ SDS and protease $\mathrm{K}$.

\section{DNA knotting by T4 topoisomerase II}

Knotting reactions contained supercoiled DNA at $\sim 20 \mu \mathrm{g} / \mathrm{ml}$ in topoisomerase II buffer $(50 \mathrm{mM}$ Tris- $\mathrm{HCl}$ at $\mathrm{pH} 7.5,60 \mathrm{mM} \mathrm{KCl}$, $40 \mu \mathrm{g} / \mathrm{ml} \mathrm{BSA}, 10 \mathrm{~mm} \mathrm{MgCl}_{2}, 0.1 \mathrm{~mm}$ EDTA). Knotting with T4 topoisomerase II was for $3 \mathrm{~min}$ at $30-32^{\circ} \mathrm{C}$, and samples were DNase I-nicked on ice as described. T4 topoisomerase II was kindly supplied by H. Benjamin.

\section{Acknowledgments}

We thank our colleagues in the Genetics Department for provision of scientific, technical, and secretarial services. We also thank our scientific colleagues in Glasgow and in site-specific recombination laboratories elsewhere for valuable discussion and unpublished information. We thank W.M. Stark and E. Morrell for their contributions to this manuscript. The work was supported by Science and Engineering Research Council.

The publication costs of this article were defrayed in part by payment of page charges. This article must therefore be hereby marked "advertisement" in accordance with 18 USC section 1734 solely to indicate this fact.

\section{References}

Argos, P., A. Landy, K. Abremski, J.B. Egan, E. Haggard-Ljungquist, R.H. Hoess, M.L. Kahn, B. Kalionis, S.V.L. Narayana, L.S. Pierson, N. Sternberg, and J.M. Leong. 1986. The integrase family of site-specific recombinases: Regional similarities and global diversity. EMBO /. 5: 443-450.

Austin, S., M. Ziese, and N. Sternberg. 1981. A novel role for site-specific recombination in maintenance of bacterial replicons. Cell 25: 729-736.

Benjamin, H.W. and N.R. Cozzarelli. 1986. DNA-directed synapsis in recombination; slithering and random collision of sites. Proc. Robert A. Welch Found. Conf. Chem. Res. 29: $107-126$.

- 1988. Isolation and characterization of the $\operatorname{Tn} 3$ resolvase synaptic intermediate. EMBO /. 7: 1897-1905.

- 1990. Geometric arrangements of $\operatorname{Tn} 3$ resolvase sites. I. Biol. Chem. 165: 6441-6447.

Boissy, R and C.R. Astell. 1985. An E. coli rec $B C$ sbcB recF host permits the deletion-resistant propagation of plasmid clones containing the $5^{\prime}$-terminal palindrome of minute virus of mice. Gene 35: 179-185.

Boocock, M.R., J.L. Brown, and D.J. Sherratt. 1986. Structural and catalytic properties of specific complexes between $\operatorname{Tn} 3$ resolvase and the recombination site res. Biochem. Soc. Trans. 14: 214-216.

_- 1987 . Topological specificity in $\operatorname{Tn} 3$ resolvase catalysis. UCLA Symp. Mol. Cell. Biol. 47: 703-718.

de Massy, B., L. Dorgai, and R.A. Weisberg. 1989. Mutations of the phage $\lambda$ attachment site alter the directionality of resolution of Holliday structures. EMBO /. 8: 1591-1599.

Droge, P. and N.R. Cozzarrelli. 1989. Recombination of knotted substrates by Tn3 resolvase. Proc. Natl. Acad. Sci. 86: $6062-6066$.

Fried, M. and D.M. Crothers. 1981. Equilibria and kinetics of lac repressor-operator interactions by polyacrylamide gel electrophoresis. Nucleic Acids Res. 9: 6505-6525.

Futcher, A.B. 1986. Copy number amplification of the 2 micron circle plasmid of Saccharomyces cerevisiae. I. Theoret. Biol. 119: $197-204$

Glasgow, A.C., K.T. Hughes, and M.I. Simon. 1989. Bacterial DNA inversion systems. In Mobile DNA led. D. Berg and M. Howel, pp. 637-759. American Society for Microbiology, Washington, D.C.

Grindley, N.D.F., M.R. Lauth, R.G. Wells, R.J. Wityk, I.J. Salvo, and R.R. Reed. 1982. Transposon-mediated site-specific recombination: Identification of three binding sites for resolvase at the res sites of $\gamma \delta$ and Tn3. Cell 30: 19-27.

Hatfull, G.F. and N.D.F. Grindley. 1988. Resolvases and DNA invertases: A family of enzymes active in site-specific recombination. In Genetic recombination /ed. R. Kucherlapati and G.R. Smith), pp. 357-396. American Society for Microbiology, Washington, D.C.

Heffron, F. and B.J. McCarthy. 1979. DNA sequence analysis of the transposon Tn3: Three genes and three sites involved in transposition of Tn3. Cell 18: 1153-1168.

Henikoff, S. 1984. Unidirectional digestion with exonuclease III creates targeted breakpoints for DNA sequencing. Gene 28: $351-359$.

Hoess, R.H., A. Wierzbicki, and K. Abremski. 1986. The role of the lox $P$ spacer region in $\mathrm{Pl}$ site-specific recombination. Nucleic Acids Res. 14: 2287-2300.

Iohnson, R.C. and M.I. Simon. 1985. Hin-mediated site-specific recombination requires two 26 -bp recombination sites and a 60-bp recombinational enhancer. Cell 41: 781-791.

Kahmann, R., F. Rudt, C. Koch, and G. Mertens. 1985. G inversion in bacteriophage $\mathrm{Mu}$ DNA is stimulated by a site within the invertase gene and a host factor. Cell 41: 771780 .

Kanaar, R., P. van de Putte, and N.R. Cozzarelli. 1989. Gin-mediated recombination of catenated and knotted DNA substrates: Implications for the mechanism of interaction between cis-acting sites. Cell 58: 147-149.

Kanaar, R., A. Klippel, E. Shekhtman, J.M. Dungan, and R. Kahmann. 1990. Processive recombination by the phage $\mathrm{Mu}$ Gin system: Implications for the mechanisms of DNA strand exchange, DNA site alignment, and enhancer action. Cell 62: $363-366$. 
Kitts, P.A. and H.A. Nash. 1988. Bacteriophage lambda site-specific recombination proceeds with a defined order of strand exchanges. I. Mol. Biol. 204: 95-107.

Kitts, P.A., L.S. Symington, P. Dyson, and D.J. Sherratt. 1983. Transposon-encoded site-specific recombination: Nature of the $\operatorname{Tn} 3$ sequences which constitute the recombination site res. $E M B O$ /. 2: 1055-1060.

Klippel, A., K. Cloppenborg, and R. Kahmann. 1988. Isolation and characterisation of unusual gin mutants. EMBO I. 7: 3983-3989.

Koch, C. and R. Kahmann. 1986. Purification and properties of the $E$. coli host factor required for inversion of the $G$ segment in bacteriophage Mu. J. Biol. Chem. 261: 1567315678.

Krasnow, M.A. and N.R. Cozzarelli. 1983. Site-specific relaxation and recombination by the $\mathrm{Tn} 3$ resolvase: Recognition of the DNA path between orientated sites. Cell 32: 13131324.

Landy, A. 1989. Dynamic, structural and regulatory aspects of site-specific recombination. Annu. Rev. Biochem. 58: 913949.

Lewis, S.M., J.E. Hesse, K. Mizuuchi, and M. Gellert. 1988. Novel strand exchanges in V(D) recombination. Cell 55: $1099-1107$.

Lewis, S. and M. Gellert. 1989. The mechanism of antigen receptor gene assembly. Cell 59: 585-588.

Nash, H.A. and C.A. Robertson. 1989. Heteroduplex substrates for bacteriophage lambda site-specific recombination: Cleavage and strand transfer products. EMBO /. 8: $3523-$ 3533.

Nunes-Duby, S.E., L. Matsumoto, and A. Landy. 1989. Half-att site substrates reveal the homology independence and minimal protein requirements for productive synapsis in lambda excisive recombination. Cell 59: 197-206.

Reed, R.R. 1981. Transposon-mediated site-specific recombination: A defined in vitro system. Cell 25: 713-719.

Reed, R.R. and N.D.F. Grindley. 1981. Transposon-mediated site-specific recombination in vitro: DNA cleavage and protein-DNA linkage at the recombination site. Cell 25: 721728.

Sadowski, P. 1986. Site-specific recombinases: Changing partners and doing the twist. J. Bacteriol. 165: 341-347.

Salvo, J.J. and N.D.F. Grindley. 1988. The gamma-delta resolvase bends the res site into a recombinogenic complex. EMBO I. 7: 3609-3616.

Sherratt, D.J. 1989. Tn3 and related transposable elements: Site-specific recombination and transposition. In Mobile DNA (ed. D. Berg and M. Howe), pp. 163-184. American Society for Microbiology, Washington D.C.

Spengler, S.J., A. Stasiak, and N.R. Cozzarelli. 1985. The stereostructure of knots and catenanes produced by phage lambda integrative recombination: Implications for mechanism and DNA structure. Cell 42: 325-334.

Stark, W.M., D.J. Sherratt, and M.R. Boocock. 1989. Site-specific recombination by $\mathrm{Tn} 3$ resolvase: Topological changes in the forward and reverse reactions. Cell 58: 779-790.

Summers, D.K. and D.J. Sherratt. 1984. Multimerization of high copy number plasmids causes instability: ColEl encodes a determinant essential for plasmid monomerization and stability. Cell 36: 1097-1103.

Sutcliffe, J.G. 1979. Complete nucleotide sequence of the Escherichia coli plasmid pBR322. Cold Spring Harbor Symp. Quant. Biol. 43: 77-90.

Wasserman, S.A. and N.R. Cozzarelli. 1985. Determination of the stereostructure of the product of $\operatorname{Tn} 3$ resolvase by a general method. Proc. Natl. Acad. Sci. 82: 1079-1083.
Wasserman, S.A., J.M. Dungan, and N.R. Cozzarelli. 1985. Discovery of a predicted knot substantiates a model for site-specific recombination. Science 229: 171-174.

Wells, R.G. and N.D.F. Grindley. 1984. Analysis of the $\gamma \delta$ res sites required for site-specific recombination and gene expression. J. Mol. Biol. 179: 667-687.

Yanisch-Perron, C., J. Vieira, and J. Messing. 1985. Improved M13 phage cloning vectors and host strains: Nucleotide sequences of the $\mathrm{Ml3mpl} 8$ and pUC19 vectors. Gene 33: $103-119$. 


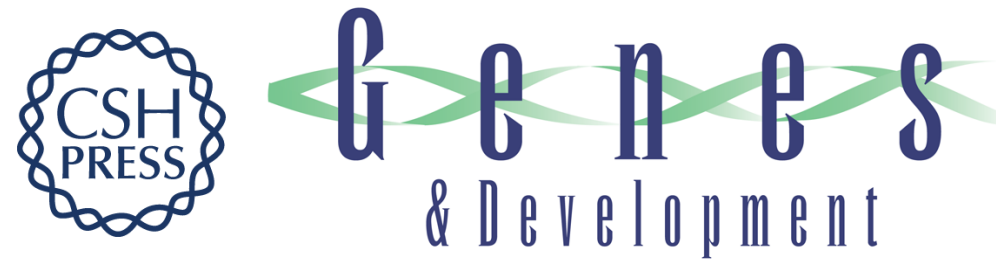

\section{Determinants of correct res site alignment in site-specific recombination by $\mathrm{Tn} 3$ resolvase.}

A L Bednarz, M R Boocock and D J Sherratt

Genes Dev. 1990, 4:

Access the most recent version at doi:10.1101/gad.4.12b.2366

References This article cites 42 articles, 7 of which can be accessed free at:

http://genesdev.cshlp.org/content/4/12b/2366.full.html\#ref-list-1

License

Email Alerting

Service

Receive free email alerts when new articles cite this article - sign up in the box at the top right corner of the article or click here.

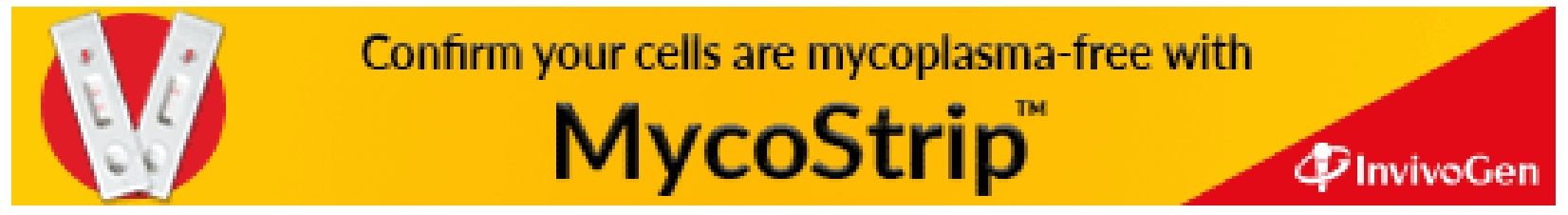

